\title{
Understanding User's Search Behavior towards Spiky Events
}

\author{
Tehran, Iran \\ Ricardo Campos \\ Polytechnic Institute of Tomar \\ Smart Cities Research Center \\ LIAAD - INESC TEC \\ Portugal \\ ricardo.campos@ipt.pt
}

Behrooz Mansouri

Web Azma Lab, Information Technology Faculty, Iran

Telecommunication Research Center

b.mansouri@itrc.ir

\author{
Mojgan Farhoodi \\ Web Azma Lab, Information \\ Technology Faculty, Iran \\ Telecommunication Research Center \\ Tehran, Iran \\ farhoodi@itrc.ir
}

\author{
Mohammad Sadegh Zahedi \\ Web Azma Lab, Information Technology Faculty, Iran \\ Telecommunication Research Center \\ Tehran, Iran \\ s.zahedi@itrc.ir
}

\author{
Maseud Rahgozar \\ Database Research Group, Control and Intelligent \\ Processing Center of Excellence, School of Electrical \\ and Computer Engineering, University of Tehran \\ Tehran, Iran \\ rahgozar@ut.ac.ir
}

\begin{abstract}
Web searches are done by users every day on a million-daily basis. Many of these web searches are related to events, social occasions that attracts society's attention. Events may happen multiple times on cyclic or non-periodic occasions. These are known as spiky events. When these events occur, multiple spikes can be observed in query logs triggered by a change in the user's behaviour and an increase in the frequency of the user's queries. In this paper, we aim to understand the user's search behaviour towards this kind of events. To this regard, we propose a new taxonomy of spiky events which categorizes queries into two groups: periodic (ongoing, historical, traditional) and aperiodic (predictable and unpredictable), and study how various features concerning the query and the clicked web pages describe the user's behaviour, before, during, and after the event. To conduct this research, we consider 100 spiky events and rely on a two-year Persian search engine query log to analyse their related queries and associated information. The results obtained show that users have a different behaviour regarding the query frequency, length and temporality, depending on the category of the spiky event and that query formulation and clicked pages are also different for each category before, during and after the event. Understanding these user's behaviours and their relationship with the different categories may play an important role for any search engine looking to provide better services for their users.
\end{abstract}

\section{ACM Reference Format}

Behrooz Mansouri, Mohammad Sadegh Zahedi, Ricardo Campos, Mojgan Farhoodi, Maseud Rahgozar. 2018. Understanding User's Search Behavior towards Spiky Events. In WWW'18 Companion: The 2018 Web Conference, April 23-27, 2018, Lyon, France. ACM, NY, NY, USA, 6 pages. https://doi.org/10.1145/3184558.3191638

This paper is published under the Creative Commons Attribution-NonCommercialNoDerivs 4.0 International (CC BY 4.0) license. Authors reserve their rights to disseminate the work on their personal and corporate Web sites with the appropriate attribution.

WWW'18 Companion April 23-27, 2018, Lyon, France.

(C) 2018 IW3C2 (International World Wide Web Conference Committee), published under Creative Commons CC BY 4.0 License.

ACM ISBN 978-1-4503-5640-4/18/04. DOI: https://doi.org/10.1145/3184558.3191638

\section{KEYWORDS}

Query Dynamics, Query Log Analysis, Temporal Queries

\section{INTRODUCTION}

Over the last few years the number of users, the volume of queries and the high number of different devices used to seek for information has increased at an incredible pace. With so many potential "clients" on the web, it was just a matter of time, for enterprises, media outlets and content providers to start publishing more and more information on the web, as way to answer their users' demands, who constantly seek for information regarding events. When talking about events, this means that users will be querying for up-to-date news, ongoing discussions, past or upcoming realizations. These events may concern different topics, going from political (e.g. elections), to sports (e.g. Olympics), holidays (e.g. Christmas) or natural disasters (e.g. earthquakes), triggering the user's attention and eventually leading to rapid increases in frequency of their related queries. One illustrative example of this may be given for the "United States presidential election" which raised a lot of attention during the month of November 2016. This can be easily observed in Figure 1 which portrays a spike of the queries issued on Parsijoo ${ }^{1}$ Persian search engine related to this event during the period 2016 - 2017. By looking at the figure, one can easily conclude that a massive interest on this topic emerged during the referred time-period as a result of an increase in the number of queries issued by the users.

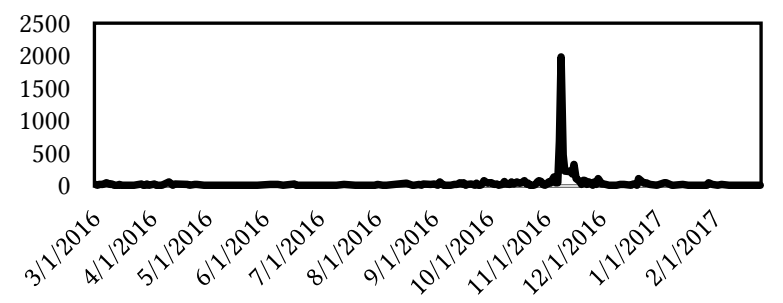

Figure 1: Query frequency pattern for queries regarding "US presidential election" from Parsijoo search engine from March 2016 to March 2017.

Several other changes in the user's behavior may be observed during the event time, including changes in query formulation, use of temporal expressions or most clicked web pages. Following our

\footnotetext{
${ }^{1}$ www.parsijoo.ir
} 
example, during the United States presidential election week, queries related to this event were 2.5 terms longer than on the week before. Also $12.5 \%$ of the related queries associated to this event contained temporal expressions such as "2016", which indicates that users were looking for information concerning this event. Moreover, most of the clicked pages during the election week also concerned the 2016 election. However, a year before the event, most clicked pages were static pages like Wikipedia which only provide general information about the event. One such change in the behavior of the user may thus affect the type of web pages retrieved by search engines (from static pages to the most recent ones). It turns out evident that understanding the users' intent and their temporal query dynamics is thus, an essential step to develop and have more effective web search engines in tasks such as query auto completion, relevant document ranking and relevant query suggestion.

Understanding the user's behavior towards the temporal aspects of a query is not a new problem and has been extensively studied in the literature over the last few years. In particular, Subasic et al. [19] studied the effect of query burst on web search and examined the behavior of search engine users during a query burst, compared to before and after this period. Kulkarni et al. [10] in turn, analyzed temporal dynamics of queries with the goal of understanding the relationship between queries, their associated documents and query intent change. They explored how queries, their relevant documents and query intent, changed over the course of 10 weeks using for that purpose, query log data, a daily web crawl and periodic human relevance judgments. Another strand of researches different from these of studies, aims to propose new algorithms related to the temporal aspects of queries. For instance, Zhang et al. [22] focused on detecting recurrent queries that are about events which occur at predictable intervals. A similar research work was proposed by Shokouhi [17] who used seasonality of query volume time series to detect seasonal queries. The work by Gupta and Berberich [7] describes a taxonomy of temporal classes at different granularities. Radinsky et al. [16] developed in turn, a temporal modeling framework adapted from signal processing and physics to predict temporal patterns in search behavior. Finally, Karmaker et al. [9], examined the influence of real world events on user queries. Given a description of an event, they identify a number of queries from search logs and characterize the temporal trend of influence created by the same event on user queries. A fully detailed description on temporal information retrieval applications can be found in the survey of Campos et al. [5].

In this paper, we go a step ahead on this kind of studies by analyzing users' behavior toward spiky events under a new taxonomy, which we believe better describes this kind of events. Similarly to previous researches $[7,10,17,22]$, we begin by dividing spiky events into two broad categories; periodic and non-periodic. The periodic events (also referred to as seasonal queries [17]) are characterized by periodic spikes in their query volumes, and happen on a regular basis, say for example, every once in a year. Instead, the non-periodic events (also known as Aperiodic) are characterized by taking place on unspecified times. Unlike related work, we break down each of these categories into further additional subcategories for which we suspect users' search behavior is different. More to the point, periodic events were divided into three different seasonal subcategories: (1) ongoing events which concern events that in each episode a new story happens which is different from previous ones (e.g. "US Presidential Elections" - we will refer to them as "P Ongoing"); (2) historical events which shows periodic spikes because of an old event happened and on every episode users pose queries about that event (e.g. "September attacks" - we will refer to them as " $P_{\text {Commemorative") and }}$ (3) special days and traditions concerning special days and traditions (e.g. "Christmas" - we will refer to them as " $P_{\text {Special days"). }}$. The non-periodic events in turn, are divided into two different subcategories: (1) those expected to happen like "Lunar eclipse" - we will refer to them as " $A_{\text {Predictable"; }}$ and (2) those that cannot be predicted like "Earthquake" - we will refer to them as "Anpredictable". Figure 2 shows each one of these different categories.

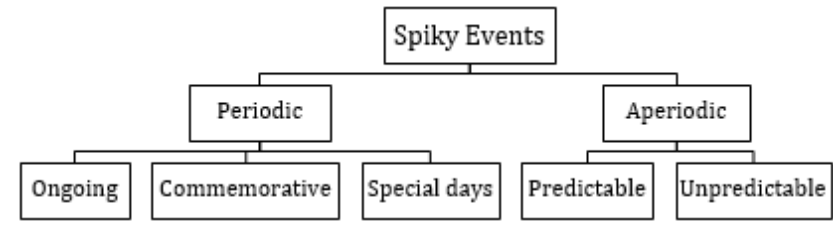

Figure 2: Spiky events categories scheme.

To study these events, we make use of query logs which provide a rich resource to understand how users react to events. For each selected event we then study a set of features related to query formulation and clicked web pages. Our contributions can be summarized as follows: (i) we examine different users' behavior toward spiky events, including the content of clicked pages, which has never been studied before; (ii) we examine these behaviors during both spike period and normal time; (iii) based on our study we prove the validity of our new taxonomy for categorizing the spiky events. The rest of this paper is structured as follows. Section 2 provides information regarding the experimental setting. Section 3, describes how users' behavior, differs, in its different facets, before, during and after the event time by examining several features from queries and clicked pages. Section 4, provides some discussion before we conclude the paper and point out some future work in Section 5.

\section{EXPERIMENTAL SETTING}

To conduct our analysis, we resort to a Persian search engine query log consisting of $27 \mathrm{M}$ queries and corresponding users' interaction with the search engine. Persian is an Indo-European language which is one of the dominant languages in the Middle East that is spoken in several countries like Iran, Tajikistan and Afghanistan. Many researches in NLP and information retrieval concern this language $[1,20,21]$. In this research, we have considered 100 spiky events selected among famous events that took place during the two-year period for which we have logs (March 2015 - March 2017). In particular, we selected 20 events for each of the 5 sub-categories, thus giving rise to the selection of 60 periodic events and 40 non-periodic. These events cover a wide range of topics, including sport events like "Olympics", political events like "United States presidential election", special days like "Christmas" or natural disasters like "Earthquake". Many of these events took place in a day while some of them occurred during multiple days. To extract queries for each event, we used the same approach as Beitzel et al. [2]. In this work, editors were asked to manually construct a list of phrases for each topic, where a topic is a set of predefined categories, from Entertainment and Shopping to Sports and Health. Instead, in our study, we asked 7 graduate students to generate a list of keywords that they would eventually use to search the 100 events. Each student was allowed to mention up to 5 keywords that he/she may use to search about a particular 
event. All the 7 lists are then intersected to avoid overlapping, thus resulting into a unique list of keywords for each event considered. Table 1 shows a few examples of keywords for the "Ballon d'Or", "Valentine" and "September $11^{\text {th }}$ " events (these terms were translated from Persian to English). Each keyword is considered a query and matched against any possible equal query registered in the logs to extract related information, such as user's query and clicked web page which will be used in our analysis. Overall, we have 100 events ( 20 per each of the 5 sub-categories considered), 63,841 queries and 23,617 clicked pages, which will be investigated in our research.

Table 1: Example of keywords lists for spiky events.

\begin{tabular}{|c|c|}
\hline Event Name & Keywords \\
\hline Ballon d'Or & Ballon d'Or, FIFA world player of the year, \\
\hline Valentine & Valentine, Valentino, The day of Love \\
\hline September 11th & September 11, 9/11, al-Qaeda attacks to US \\
\hline
\end{tabular}

\section{EXPERIMENTAL RESULTS}

In this section we will present our experimental results. First, we will present the results regarding changes on the query volume frequency (Section 3.1), query length (Section 3.2) and use of temporal expressions (Section 3.3). Next, we will investigate how users' clicks vary for each of the spiky events by considering both diversity of clicked pages (Section 3.5) and pages content (Section 3.6).

\subsection{Query Frequency}

Our first analysis is done on query volume changes. Our aim is to understand how the frequency of queries related to spiky events change before, during and after the event. In addition, we are also interested to understand whether there is any substantial difference regarding the number of queries issued before, during and after the event time. This can be very useful for search engines aiming to provide better query suggestions and auto completions. To track changes in query volume we define four different time frames. Event frame $(\mathrm{EF})$ is the time when the event takes place. For some events like "Oscar" we considered one day as event frame, while for other events like "Olympics" we consider its event frame from 5 August to 21 August 2016. The Pre-Event frame ( $\mathrm{pre} E \mathrm{EF}$ ) is simply defined as the whole week before the Event frame (EF), while the Post-Event frame $\left(\mathrm{EF}_{\mathrm{post}}\right)$ is the whole week after the Event frame (EF). Finally, the Event window (EW) is the total duration of these three frames. Other times are considered as Normal window (NW). As we are investigating a two-year query log we considered the average amount of queries, as events like "Valentine" and "Oscar", may occur more than once during this two time-period. Table 2 presents the average percentage of queries that were submitted to the search engine, for each of the 5 categories and of the 20 spiky events considered.

Overall, more than $57.9 \%$ of the queries $(62.07 \%$ on average) have been submitted to the search engine during the event window (EW) for all the types of spiky events. If only Periodic events are considered, one can observe that when looking at the Post-Event frame, a considerable difference between $P_{\text {Ongoing }}$ related queries (23.16\%), $P_{\text {Comemorative }}(10.11 \%)$ and $P_{\text {Special days }}(5.31 \%)$ can be noticed. The main reason is that $P_{\text {Ongoing }}$ events do usually result into some future consequences eventually leading users to get information about them. For example, after the "American presidential election" many queries were submitted to the search engine to gain information about the election winner and on how people voted for the candidates on the different states.

Table 2: Average percentage of queries submitted to the search engine regarding each class of spiky events in each time frame.

\begin{tabular}{|c|c|c|c|c|c|c|}
\hline & Event Type & $\mathrm{pre}^{\mathrm{EF}}$ & $\mathrm{EF}$ & $\mathrm{EF}_{\text {post }}$ & EW & NW \\
\hline \multirow{3}{*}{ 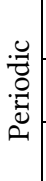 } & $\mathrm{P}_{\text {OnGoing }}$ & $7.56 \%$ & $32.51 \%$ & $23.16 \%$ & $63.23 \%$ & $36.77 \%$ \\
\hline & $\mathrm{P}_{\text {SpecialDays }}$ & $38.14 \%$ & $26.77 \%$ & $5.31 \%$ & $70.22 \%$ & $29.78 \%$ \\
\hline & $\mathrm{P}_{\text {Commemorative }}$ & $30.64 \%$ & $17.47 \%$ & $10.11 \%$ & $58.23 \%$ & $41.77 \%$ \\
\hline \multirow{2}{*}{ 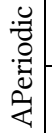 } & A $_{\text {Predictable }}$ & $8.64 \%$ & $15.32 \%$ & $33.94 \%$ & $57.90 \%$ & $42.10 \%$ \\
\hline & $\mathrm{A}_{\text {UnPredictable }}$ & $1.29 \%$ & $13.90 \%$ & $45.58 \%$ & $60.77 \%$ & $39.23 \%$ \\
\hline
\end{tabular}

In contrast, $P_{\text {Special days }}$ and $P_{\text {Comemorative }}$ events, are mostly submitted during the Pre-Event frame ( ${ }_{\mathrm{pre}} \mathrm{EF}$ ) with $38.14 \%$ and $30.64 \%$ respectively. And, while there is a drop during the event frame (EF), it only turned out to be more evident after the event $\left(\mathrm{EF}_{\text {post }}\right)$. Another interesting thing is that the percentage of submitted queries in the Post-Event frame $\left(\mathrm{EF}_{\text {post }}\right)$ for $P_{\text {Comemorative }}$ queries, $(10.11 \%)$ is approximately twice of $P_{\text {Special days }}(5.31 \%)$ queries. This may be due to their historical origins and to the fact that this kind of events are usually officially celebrated by wellknown and famous people, thus giving rise to a number of searches where users predominantly look for news regarding their favorite celebrity. As for the Aperiodic events, one can notice that their frequency is noticeably higher in the Post-Event frame $\left(\mathrm{EF}_{\text {post }}\right)$ when compared to Periodic events. For instance, for $A_{\text {Unpredictable }}$ queries users have no idea that the event may be occurring. The $1.29 \%$ of queries that are recorded in Pre-Event frame ( $\mathrm{pre}_{\mathrm{EF}}$ ) regards some of these events that have happened (e.g., "Bam earthquake") and for which people search on their anniversary. In the case of $A_{\text {Predictable }}$ queries, users tend to know more about upcoming events and therefore have a tendency to pose more queries during the Pre-Event frame $\left({ }_{\mathrm{pre}} \mathrm{EF}\right)$ when compared to $A_{\text {Unpredictable }}$. Yet, most of the queries are issued during the Post-Event frame $\left(\mathrm{EF}_{\text {post }}\right)$. Another important thing to note is that despite most of the Aperiodic events takes place in a single day, still several users continue to look for information related to the event in the coming days. This could be easily observed for the "Paris terrorist attacks" event, which saw a huge increase in the number of queries posed after the Event frame $\left(\mathrm{EF}_{\text {post }}\right)$, with several users looking for information related to this tragic event, such as the number of people killed or injured, who was responsible for the attack, what happened to the terrorists or how France political parties reacted to this attack.

\subsection{Query Length}

After examining query frequency, we will now have a look at query length. Our aim is to study the distribution of query length for each type of spiky event as several experiments [3,4,6,11] have proven that existing retrieval methods, perform worse, in general, for long queries than for short ones. In addition, we also aim to provide information on the distribution of query length with regard to other types of queries (that are not specifically about spiky events, such as "computer science", "weather condition" or "electronic books"). Our aim is to study how spiky events queries 
are different from other queries in terms of query length. For this, we randomly selected 500.000 queries during the period of 6 months, outside the frame of all the other periodic and aperiodic events. Figure 3 shows the boxplot of the distribution of the queries for each category of spiky events. Other queries are labeled as Other.

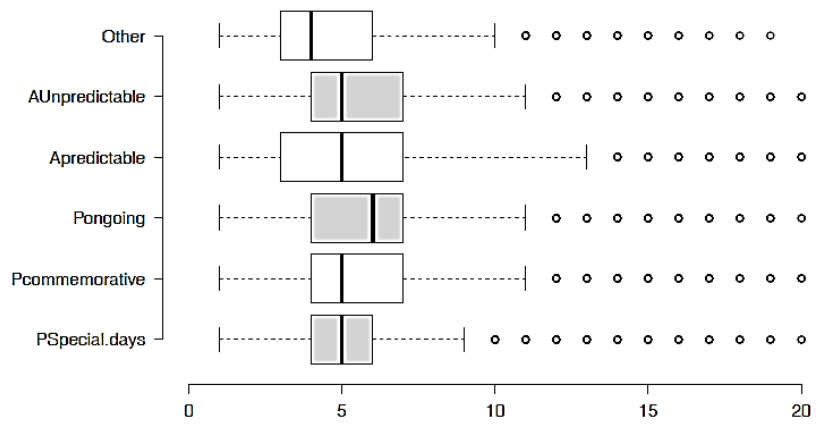

Figure 3. Boxplot of query length distribution for each of spiky event category.

As can be seen from this figure, the query length distribution is nearly the same for all categories, with most frequent queries having length between 4 and 6 terms, thus suggesting a rightskewed distribution, with a lot of queries having a few terms and a few queries having a lot of terms. Note however, that queries for $P_{\text {Ongoing }}$ events were longer than the other query categories with 6.1 terms per query on average. This may be understood as each episode has its own several interesting happenings. For example, for events such as "Olympics", people often tend to issue longer queries during the event time to get the exact news that they are looking for. From Figure 3 we can also observe that the frequency of spiky events queries drops gradually from 6 terms onward with queries with length more than 13 becoming a rarity. However, compared to other types of queries this slope is slower. Another important result is that the average query length for spiky events was 5.7 terms per query, 3.5 terms higher than the average number of terms used in general web queries which was reported by Jansen et al. [8] to be 2.2 terms. The experiment conducted also shows that queries related to spiky events contain on average 2.2 terms more than other types of Persian queries [1] which shows that in general, Persian queries are longer than English queries, but queries related to spiky events are even longer.

\subsection{Use of Temporal Expressions in Queries}

One of the characteristics of spiky events is that they are time dependent. Usually this is expressed by the users with resort to explicitly or implicitly temporal information needs who issue queries with different temporal expressions. The use of both implicit and explicit temporal expressions is a well-studied problem in the literature $[7,14,18]$. In this study, we aim to understand how temporality explicitly affects the different types of spiky events (e.g., bam earthquake 2003). To this purpose, we used our recently developed Persian time tagger tool, ParsTime [12], and found that for spiky event queries, $23.7 \%$ of the total queries contain temporal expressions $(21.5 \%$ when considering only distinct queries (considering queries not their frequency)) which is noticeably higher than the $1.5 \%$ stated by Nunes et al. [15] for general queries. This confirms that queries related to spiky events are undoubtfully temporal dependent. Overall, we have found that queries regarding Periodic events contain more temporal expressions when compared to Aperiodic events. For instance, $P_{\text {Ongoing }}$ queries contained the most temporal expressions with an average number of $39.5 \%$ queries for each event. The main reason is that a $P_{\text {Ongoing }}$ event has a unique name, leading users to refer to it every time it occurs. For example, different episodes of "Fifa world cup" are referred to as "Fifa world cup 2018", "Fifa world cup 2014" or "Fifa world cup 2010". We could also confirm that $24.8 \%$ of the queries related to $P_{\text {Comemorative }}$ events were used with temporal expressions, but when considering distinct queries, only $8.87 \%$ of the queries contained temporal expressions. Recall that this type of spiky event refers to historic realizations taking place in one unique time and for which users mostly use the temporal expression related to that unique time. In contrast Periodic events, Aperiodic had the lowest amount of temporal expressions in their queries with only $10.3 \%$ and 13.5 for $A_{\text {Predictable }}$ and $A_{\text {Unpredictable }}$ events respectively. One possible explanation for this is that users tend to only refer to the name of the event or the place in which the event took place. Figure 4 gives information on the percentages of queries with temporal expressions considering both total and distinct queries for each category of events.

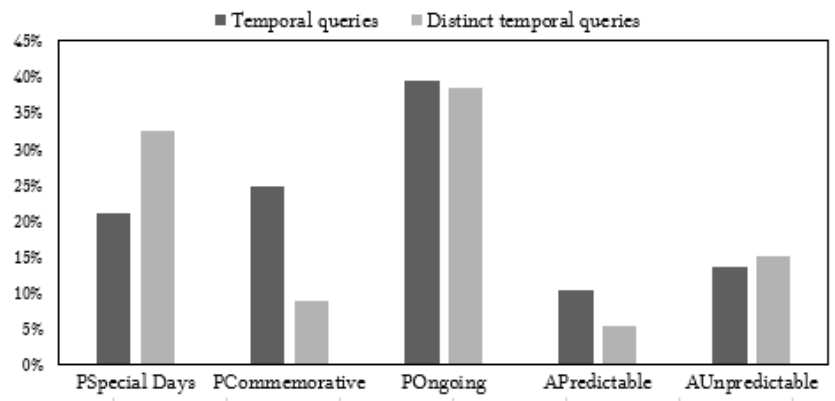

Figure 4: Percentage of total and distinct queries regarding spiky events containing temporal expressions.

To further understand these results, we distribute each query with a temporal expression in each of the time-frames introduced in Section 3.1. Final results are presented in Table 3.

Table 3: Distribution of queries with temporal expression according to the defined time frames.

\begin{tabular}{|c|c|c|c|c|c|c|}
\hline & Event Type & ${ }_{\text {pre }} \mathrm{EF}$ & $\mathrm{EF}$ & $\mathrm{EF}_{\text {post }}$ & EW & NW \\
\hline \multirow{3}{*}{ 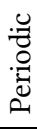 } & $\mathrm{P}_{\text {OnGoing }}$ & $7.5 \%$ & $10.4 \%$ & $21.7 \%$ & $39.6 \%$ & $60.4 \%$ \\
\hline & $\mathrm{P}_{\text {SpecialDays }}$ & $35.9 \%$ & $23.4 \%$ & $10.7 \%$ & $70.0 \%$ & $30.0 \%$ \\
\hline & $\mathrm{P}_{\text {Commemorative }}$ & $5.4 \%$ & $4.8 \%$ & $9.8 \%$ & $20.0 \%$ & $80.0 \%$ \\
\hline \multirow{2}{*}{ 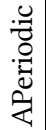 } & A Predictable & $13.2 \%$ & $2.6 \%$ & $30.0 \%$ & $45.8 \%$ & $54.2 \%$ \\
\hline & $\mathrm{A}_{\text {UnPredictable }}$ & $0.0 \%$ & $0.0 \%$ & $24.5 \%$ & $24.5 \%$ & $75.5 \%$ \\
\hline
\end{tabular}

As can be observed from this table, the use of temporal expressions in queries differs a lot depending on the category. For $P_{\text {Ongoing }}$ events, $60.4 \%$ of these queries were issued, on average, during the Normal window (NW). During the event time (EF), users tend to simply ask about the event in the expectation that the search engine returns relevant results of it. Instead, in Normal window (NW) they prefer to specify which episode of the event they are referring to. For instance, $71.3 \%$ of the queries with temporal expressions regarding the event "Oscar" contained year 
expressions that specified which episode of "Oscar", users are referring to. Another interesting result is that $70 \%$ of the queries with temporal expressions for $P_{\text {Special days }}$ events were posed to the search engine during the Event window (EW) mostly concerning the current events and seeking information about how to celebrate and commemorate the special day. For example, temporal expressions 1394 and 1395 (which indicates the Persian years for which, the query $\log$ is available, i.e., 2015 and 2016 respectively) constitute $78.2 \%$ of the queries with temporal expressions regarding the event "Valentine". Another important result is that for $P_{\text {Comemorative }}$ events, $80 \%$ of the temporal expressions were used during the Normal window (NW) which indicates that during the event window (EW) less temporal expressions are used. Considering Aperiodic events, for $A_{\text {Unpredictable }}$ category, queries with temporal expressions during Event window (EW) mostly concerned the recent event. However, for both $A_{\text {Unpredictable }}$ and $A_{\text {Predictable }}$ categories, some of the temporal expressions used during the Event window (EW) were posed regarding the past episodes. That is, users tend to also gain information about the previous events. For example, after the "Paris terrorist attack" event, users also search for other terrorist attacks like "London bombing 2005" or "2004 Madrid train bombing". Temporal expressions used for these two types of events during Normal window (NW) concerned different episodes of the event. Moreover, the years in which events with higher impacts and consequences took place were more mentioned by users. For example, $58.9 \%$ of the queries with temporal expressions that concerned the event "Earthquake" were mentioning the year 2003 when the most destructive earthquake of recent years took place in Bam city.

\subsection{Diversity of Clicked Web Pages}

In this section, we aim to study the variability of clicked pages to understand if there is any different effect due to the diverse types of spiky queries. For that, we resort to click entropy. Low click entropy indicates that users prefer a few unique URLs regarding the event, while higher click entropy shows a wide range of URLs were targeted by users. The relation between query popularity and click entropy was studied in [10]. They reported that queries with multiple spikes have higher click entropy compared to queries with only one spike and assumed this was due to generation of a new query intent in each spike for the same query. In this research, we calculate click entropy for each type of spiky events and also to compare click entropy during Event and Normal window. Click entropy for an event is calculated as follows:

lick Entropy $(E)=-\sum_{U R L u}(P(u \mid E)) \times\left(\log _{2} P(u \mid E)\right)$

Where $P(u \mid E)$ is the probability that the URL $\mathrm{u}$ is clicked by the users when they are searching about the event E. Resorting to this equation, we calculated the average click entropy for spiky events. The results obtained show that the average click entropy for spiky events is 6.21, thus suggesting a high diversity between clicked web pages. The highest click entropy belongs to $P_{\text {Special days }}$ category with 7.17 while $A_{\text {Unpredictable }}$ events, with 5.21, have the lowest. To provide a better insight in what concerns to the diversity of clicked web pages over time, we also measured click entropy during the Event window (EW) and the Normal window (NW). Our aim was to study whether there is any substantial different behavior between the time that refers to the total duration of the event as opposed to the remaining times. The results obtained are presented in Figure 5.

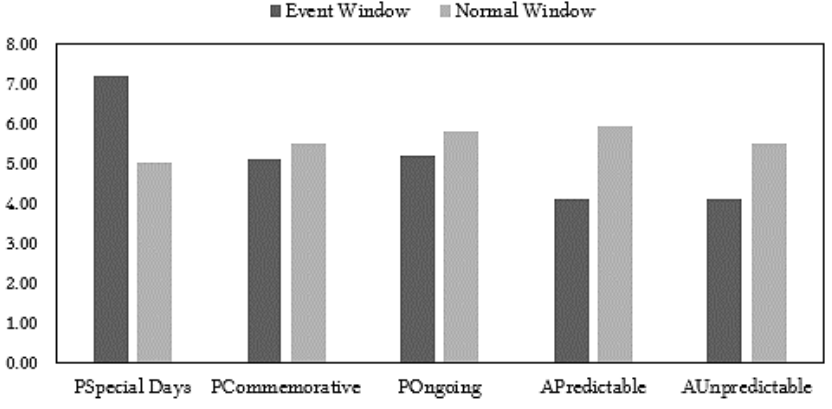

Figure 5: Average click entropy of spiky event categories during Event and Normal window.

By looking at the figure, one can notice that for $P_{\text {Special days }}$ events, click entropy was noticeably higher during the Event window (EW) when compared to the Normal window (NW). Actually, this was the only type for which click entropy was higher during the Event window (EW). Indeed, for any other types, click entropy was always higher for Normal window (NW). A detailed analysis on the remaining types, enables to conclude that $P_{\text {Ongoing }}$ and $P_{\text {Comemorative }}$ events, had approximately the same click entropy, while for $A_{\text {Predictable }}$ and $A_{\text {Unpredictable }}$ event click entropy was lower during the Event window (EW). During the Event window (EW), for both types of Aperiodic events, click entropy is lower compared to Normal window (NW), as users mostly prefer on recent pages. Based on these results, we can conclude that regardless the type of the Periodic event, users tend to click, during the event window (EW), on more pages when compared to Aperiodic events. Still, further features, such as the content of web pages, need to be explored in order to take more valid conclusions regarding the type of pages preferred by users during the event window (EW) and the normal window. We will have a look at this in the next section.

\subsection{Content of Clicked Web Pages}

In this last section, we analyse the content of clicked web pages. Knowing the types of pages preferred by users may play an important role in defining a more elaborated ranking strategy. To conduct this experiment, we will investigate the content of top clicked web pages. For each of the 100 events deemed, we considered the top-200 pages that were more frequently clicked by users (100 pages from the Event window and 100 from the Normal window) totalizing 20.000 web pages. We then asked three students to look at the content of each web page and to manually classify them with regard to recency, oldness and Wikipedia-like page (a type of navigational page that is usually retrieved for events). Each web page is classified into: (1) Recent Pages; which provide information about the most recent episode of the event, (2) Wikipedia-like Pages; which gives general information about the event and (3) Old Pages; which concerns the old episodes of the event. Our experiments on query logs show that the results to be retrieved differ during the Event window and the Normal window for each event category class. Table 4 summarizes the results of our study, by showing the percentage of clicked pages per page category during peak and non-peak times for each event class. 
Table 4: Average percentage of clicked pages in each content category, considering type of spiky event during Event and normal.

\begin{tabular}{|c|c|c|c|c|c|c|c|}
\hline & \multirow{2}{*}{ Event Type } & \multicolumn{2}{|c|}{ Recent Page } & \multicolumn{2}{|c|}{$\begin{array}{l}\text { Wikipedia- } \\
\text { Like pages }\end{array}$} & \multicolumn{2}{|c|}{ Old Pages } \\
\hline & & EW & $v$ & EW & NW & EW & NW \\
\hline \multirow{3}{*}{ 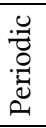 } & & & & $4.1 \%$ & $91.3 \%$ & $\%$ & $\%$ \\
\hline & $\mathrm{P}_{\text {Spec }}$ & $54.7 \%$ & $7.3 \%$ & $44.9 \%$ & $90.6 \%$ & $0.4 \%$ & $2.1 \%$ \\
\hline & $\mathrm{P}_{\mathrm{Comn}}$ & $92.1 \%$ & $14 \%$ & $2.5 \%$ & $7.1 \%$ & $5.4 \%$ & $41.5 \%$ \\
\hline \multirow{2}{*}{ 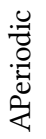 } & Pre & 20 & $4.9 \%$ & $12.6 \%$ & $77.3 \%$ & $3.6 \%$ & $7.8 \%$ \\
\hline & $A_{\text {UnPredictable }}$ & $94.2 \%$ & $34.8 \%$ & $4.2 \%$ & $35.4 \%$ & $1.6 \%$ & $29.8 \%$ \\
\hline
\end{tabular}

Observing the results for $P_{\text {Ongoing }}$ events one can conclude that during the Event window (EW), users mostly prefer recent web pages, thus retrieving more fresh documents seems to be the best choice. This contrasts with Normal window (NW), for which temporal diversity is suggested. Likewise, for the Event window (EW) on $P_{\text {Commemorative }}$ events, users are mostly interested in getting to know about recent commemorative and memorial gatherings. However, in contrast to $P_{\text {Ongoing }}$ events, a notable amount of users were also interested in Wikipedia-like pages. On Normal window (NW), Wikipedia-like pages were also dominantly clicked compared to other types of web pages. $P_{\text {Special days }}$ events were mostly favoured with recent pages during Event window (EW), while Wikipedia-like pages were preferred on Normal window (NW). Finally, for both types of Aperiodic events recent pages were mostly selected by users when considering the Event window (EW). However, a different behaviour can be observed during Normal window (NW). For $A_{\text {Predictable }}$ events Wikipedia-like pages were generally selected, but for $A_{\text {Unpredictable }}$ events temporal diversity is needed as different type of pages were equally selected by users.

\section{DISCUSSION}

Spiky events are events that take place multiple times and trigger a shift on user's demands during their occurrence. In this paper, we have studied users' behavior to spiky events from different perspectives including query and clicked pages. We have shown that users behave differently towards the 5 categories of spikes defined: ongoing events (e.g., "Olympics"), historical events (e.g., "September $11^{\text {th }}$ attacks"), special days events (e.g., "Valentine"), Aperiodic expected events (e.g., "Lunar eclipse") and unanticipated events (e.g., "Earthquake".)

To understand the changes in users' behavior with regard to event occurrence time, we defined 5 time-frames: before, during and after the event, duration of all three-time frame as Event window, and Normal window, which refers to the remaining time not in the Event window. We started our analysis by exploring the changes in query volume, query length and use of temporal expressions. Overall, we could note the occurrence of specific patterns on the user's behavior for each of the event categories. This could be leveraged by search engines to provide better services regarding query suggestion, query auto completion and result ranking. Next, we studied the diversity of the clicked web pages for each event. We could conclude that there was a higher diversity for all the types of spiky events, when compared to nonspiky ones (which we labeled as Others), and that the variety of clicked web pages for all categories, with the exception of $P_{\text {Special }}$ days, was lower during the event time when compared to other times of the event. We then analyzed the content of clicked pages by users related to spiky events into three categories: pages giving general information about the event (regarded as Wikipedia-Like pages), pages concerning the recent episodes and pages providing information about past episodes of the event. This enabled us to conclude that frequently clicked web pages are different for each class of spiky events during Event and Normal window thus suggesting the need to have different ranking approaches for each type of event with regard to the query issue time. Overall, we have proven that specific patterns can be recognized in user's behavior when their information need concerns spiky events. Typical search services such as query suggestion, query auto completion and results ranking can be eventually more efficient and increase users' satisfaction, should search engines leverage the knowledge they have about the users' behavior toward spiky events.

\section{CONCLUSIONS}

In this paper users' behavior toward spiky events were investigated. To conduct this study, we resorted to a two-year query $\log$ of a Persian search engine to study 100 spiky events (overall 1.344.700 log records) and studied how queries and clicked pages change for these events when they take place. We concluded that each type of spiky event demands its own search services. In future work, we will try to propose efficient search services for each type of spiky event. First, a method is needed to detect queries regarding the different types of spiky events. Our papers on $[13,14]$ are the first attempts on this matter. Then for each individual type of event, specific retrieval and ranking methods, query auto completion, query suggestion and summarization can be proposed.

\section{ACKNOWLEDGMENTS}

This research was supported by Persian native search engine program from Iran Tele-communication Research Center (ITRC). It was also partially funded by the ERDF through the COMPETE 2020 Programme within project POCI-01-0145-FEDER-006961, and by National Funds through the FCT as part of project UID/EEA/50014/2013.

\section{REFERENCES}

[1] AleAhmad, A., Zahedi, M., Rahgozar, M., \& Moshiri, B. (2016). irBlogs: A standard collection for studying Persian bloggers. Computers in Human Behavior, 57, 195-207.

[2] Beitzel, S. M., Jensen, E. C., Chowdhury, A., Frieder, O., \& Grossman, D. (2007). Temporal analysis of a very large topically categorized web query log. Journal of the American Society for Information Science and Technology, 58(2), 166-178.

[3] Bendersky, M., \& Croft, W. B. (2008). Discovering key concepts in verbose queries. In Proceedings of the 31st annual international ACM SIGIR conference on Research and development in information retrieval, ACM'08, 491-498.

[4] Broder, A. Z., Carmel, D., Herscovici, M., Soffer, A., \& Zien, J. (2003). Efficient query evaluation using a two-level retrieval process. In Proceedings of the twelfth international conference on Information and knowledge management, ACM'03, 426-434.

[5] Campos, R., Dias, G., Jorge, A. M., \& Jatowt, A. (2014). Survey of temporal information retrieval and related applications. ACM Computing Surveys (CSUR), 47(2), 15.

[6] Downey, D., Dumais, S., Liebling, D., \& Horvitz, E. (2008). Understanding the relationship between searchers' queries and information goals. In Proceedings of the 17th ACM conference on Information and knowledge management, ACM'08, 449-458.

[7] Gupta, D., \& Berberich, K. (2015). Temporal query classification at different granularities. In International Symposium on String 
Processing and Information Retrieval, Springer, Cham, 156-164.

[8] Jansen, B. J., \& Spink, A. (2006). How are we searching the World Wide Web? A comparison of nine search engine transaction logs. Information processing \& management, 42(1), 248-263.

[9] Karmaker Santu, S. K., Li, L., Park, D. H., Chang, Y., \& Zhai, C. (2017). Modeling the Influence of Popular Trending Events on User Search Behavior. In Proceedings of the 26th International Conference on World Wide Web Companion, International World Wide Web Conferences Steering Committee, 535-544.

[10] Kulkarni, A., Teevan, J., Svore, K. M., \& Dumais, S. T. (2011). Understanding temporal query dynamics. In Proceedings of the fourth ACM international conference on Web search and data mining, ACM'11, 167-176.

[11] Kumaran, G., \& Allan, J. (2007). A Case For Shorter Queries, and Helping Users Create Them. In HLT-NAACL, 220-227.

[12] Mansouri, B., Zahedi, M. S., Campos, R., Farhoodi, M., and Rahgozar,M. (2018). ParsTime: Rule-Based Extraction and Normalization of Persian Temporal Expressions. In ECIR'18.

[13] Mansouri, B., Zahedi, M. S.,Rahgozar,M.,and Campos, R. (2017). Detecting Seasonal Queries Using Time Series and Content Features. In ICTIR'17, 1-4.

[14] Mansouri, B., Zahedi, M. S., Rahgozar, M., Oroumchian, F., Campos, R. (2017). Learning Temporal Ambiguity in Web Search Queries. In CIKM'17, 6-10.

[15] Nunes, S., Ribeiro, C., \& David, G. (2008). Use of temporal expressions in web search. In European Conference on Information Retrieval Springer, 580-584.

[16] Radinsky, K., Svore, K. M., Dumais, S. T., Shokouhi, M., Teevan, J.,
Bocharov, A., \& Horvitz, E. (2013). Behavioral dynamics on the web: Learning, modeling, and prediction. ACM Transactions on Information Systems (TOIS), 31(3), 16.

[17] Shokouhi, M. (2011). Detecting seasonal queries by time-series analysis. In Proceedings of the 34th international ACM SIGIR conference on Research and development in Information Retrieval, ACM'17, 1171-1172.

[18] Strötgen, J., \& Gertz, M. (2010). Heideltime: High quality rule-based extraction and normalization of temporal expressions. In Proceedings of the 5th International Workshop on Semantic Evaluation, 321-324. Association for Computational Linguistics.

[19] Subasic, I., \& Castillo, C. (2010). The effects of query bursts on web search. In Web Intelligence and Intelligent Agent Technology (WIIAT), IEEE/WIC/ACM International Conference on IEEE'10, 374-381.

[20] Zahedi, M., Aleahmad, A., Rahgozar, M., Oroumchian, F., \& Bozorgi, A. (2017). Time sensitive blog retrieval using temporal properties of queries. Journal of Information Science, 43(1), 103-121.

[21] Zahedi, M. S., Mansouri, B., Moradkhani, S., Farhoodi, M., \& Oroumchian, F. (2017). How questions are posed to a search engine? An empiricial analysis of question queries in a large scale Persian search engine log. In Web Research, ICWR'17, 3th International Conference on IEEE, 84-89.

[22] Zhang, R., Konda, Y., Dong, A., Kolari, P., Chang, Y., \& Zheng, Z. (2010). Learning recurrent event queries for web search. In Proceedings of the Conference on Empirical Methods in Natural Language Processing, 1129-1139. Association for Computational Linguistics. 